\title{
Evolution of Massive Stars along the Cosmic History
}

\author{
Georges Meynet, Sylvia Ekström, Cyril Georgy, Cristina \\ Chiappini and André Maeder \\ Astronomical Observatory of the Geneva University \\ CH-1290 Versoix, Switzerland \\ georges.meynet@unige.ch
}

\begin{abstract}
Massive stars are "cosmic engines" (cf the title of the IAU Symposium 250). They drive the photometric and chemical evolution of galaxies, inject energy and momentum through stellar winds and supernova explosions, they modify in this way the physical state of the interstellar gas and have an impact on star formation. The evolution of massive stars depends sensitively on the metallicity which has an impact on the intensity of the line driven stellar winds and on rotational mixing. We can distinguish four metallicity regimes: 1.- the Pop III regime $0 \leq Z<\sim 10^{-10}$; 2.- The low metallicity regime $10^{-10} \leq Z<0.001$; 3.- The near solar metallicity regime $0.001 \leq Z<0.020$; 4.- The high metallicity regime $0.020 \leq Z$. In each of these metallicity ranges, some specific physical processes occur. In this review we shall discuss these physical processes and their consequences for nucleosynthesis and the massive star populations in galaxies. We shall mainly focus on the effects of axial rotation and mass loss by line driven winds, although of course other processes like binarity, magnetic fields, transport processes by internal waves may also play important roles.
\end{abstract}

\section{The evolution of the metallicity during the cosmic history}

In the following, we shall call metallicity the quantity $Z$ which is the mass fraction of all the elements with atomic mass number greater than that of helium. In the course of the cosmic history, due to stellar nucleosynthesis, $Z$ increases globally in the Universe, although at different paces in different environments. In Fig. [1, the evolution of $[\mathrm{Fe} / \mathrm{H}]^{1}$ as predicted by the chemical evolution model for the halo of our Galaxy of Chiappini et al. (2006b) is shown. This evolution corresponds to that expected after an intense starburst which occurred at time zero and was responsible for driving a galactic wind. One can see that ultra-metal poor stars (i.e. stars with $[\mathrm{Fe} / \mathrm{H}]$ below -4 )

\footnotetext{
${ }^{1}$ Note that $[\mathrm{Fe} / \mathrm{H}]$ defined as $\lg \left(\frac{\left(n_{F e} / n_{H}\right)_{*}}{\left(n_{F e} / n_{H}\right) \odot}\right)$, where $n_{F e}, n_{H}$ are the number density of iron, respectively of hydrogen and where the subscript $*$ indicates that the composition is that of the star considered and the subscript $\odot$ that of the Sun.
} 


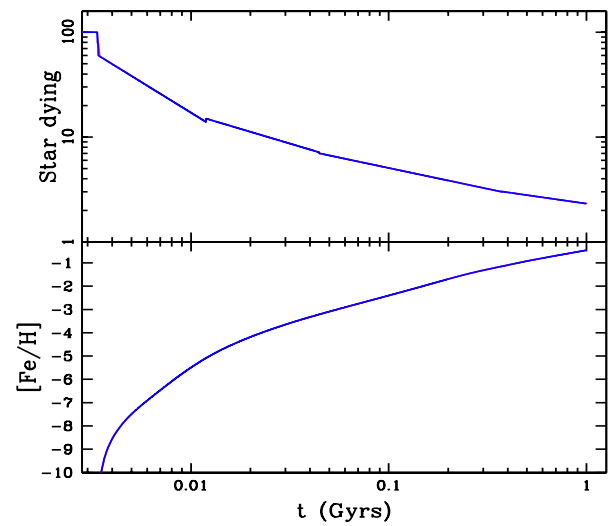

Figure 1: After a starburst having occurred at time $t=0$, the top panel shows the variation as a function of time of the minimum initial mass of stars having finished their evolution at the considered time. The bottom panel shows the evolution as a function of time of $[\mathrm{Fe} / \mathrm{H}]$. The chemical evolution model is from Chiappini et al. (2006b).

have acquired their metallicity after less than 30 Myrs. Their heavy element content comes from matter processed and ejected by stars with masses above $10 \mathrm{M}_{\odot}$.

At this stage it is interesting to note three points

- We note that metallicity increases very rapidly, indicating that the period of time during which Pop III stars dominate the nucleosynthesis is very short.

- Systems like the halo of our Galaxy gives us the chance to observe stars whose composition reflect only two specific nucleosynthetic processes: the Big Bang nucleosynthesis, and massive stars nucleosynthesis. This is in contrast with for instance solar metallicity stars in the Milky Way whose initial heavy elements abundances reflect the effects of many stellar generations and of various nucleosynthetic processes encompassing e.g. intermediate mass stars, novae and type Ia supernovae. In that case, the link between the observed (surface) composition and the stellar yields is much less straightforward than for very metal poor halo stars.

- Finally, let us mention that a low or even very low metallicity does not necessarily implies that only massive stars have contributed to the heavy elements enrichments of the stars. A very low metallicity can result from (very) low star formation rates over long periods. In that case the surface abundances of non-evolved star reflect the stellar enrichments 
having occurred under the action of stars of a much broader mass range than only massive stars. A way to discriminate between the case of a low star formation rate over long period and the one of a strong star formation rate observed at a very early stage is to look at abundance ratios (as for instance $[\alpha / \mathrm{Fe}]$, see Matteucci 2001). In the case of the halo of our Galaxy the observed $[\alpha / \mathrm{Fe}]$ ratios indicates a short timescale for the heavy elements enrichment.

The considerations above indicate that the halo of our Galaxy offers a wonderful opportunity to study the nucleosynthesis due to the first massive star generations. As we shall see below many puzzling observed facts are related to the halo stars both in the field and in the globular clusters (see Sect. 2.4 and 4 ).

\section{The effects of metallicity on radiatively driven stellar winds and on rotational mixing}

Let us recall that a change of $Z$, the mass fraction of heavy elements, also affects the initial mass fraction of hydrogen, $X$, and of helium, $Y$. When $Z$ increases, $X$ decreases and $Y$ increases. The increase of the abundance in helium with respect to $Z$ is conveniently described by a quantity, $\Delta Y / \Delta Z$, defined as the increase of helium per unit increase in $Z$. The abundance of helium at a given stage, $Y$, is given by $Y=Y_{0}+\Delta Y / \Delta Z \times Z$, where $Y_{0}$ is the primordial He abundance. A typical value for $\Delta Y / \Delta Z$ at solar metallicity is around 2 (see e.g. Casagrande et al. 2007). Recently much higher values of $\Delta Y / \Delta Z$ (of the order of 70) has been deduced from the presence in globular clusters of multi-ZAMS sequences (Piotto et al. 2005). We shall come back on that question in Sect. 4 below. Thus probably $\Delta Y / \Delta Z$ varies as a function of the metallicity and of other environmental factors.

At low metallicities $(Z \leq 0.02), Y$ remains practically constant, and the stellar properties as a function of metallicity are thus expected to be determined mainly by $Z$. At high metallicities $(Z \geq 0.02)$, on the other hand, $Y$ increases (or, alternatively, $X$ decreases) significantly with $Z$. In those conditions, both $Z$ and $Y$ (and $X=1-Y-Z$ ) determine the stellar properties as a function of metallicity.

In non rotating, non mass losing models, the metallicity affects the evolution of stars mainly through its impact on the radiative opacities, the equation of state and the nuclear reaction rates. These effects of metallicity on stellar models are discussed in details in Mowlavi et al. (1998).

For stars with initial mass greater than about $30 \mathrm{M}_{\odot}$, mass loss becomes an important ingredient already during the MS phase and the effects of metallicity on the mass loss rates have to be taken into account. Metallicity also affects the transport mechanisms induced by rotation (Maeder \& Meynet 2001). Typically a given initial mass star, starting its evolution with a given initial velocity, will be more efficiently mixed by rotation at low than at high 
metallicity. Below we explain why such a behavior is expected.

\subsection{Line driven winds at different $Z$}

The main trigger of stellar winds is radiation pressure. As written by Eddington (1926) " . . the radiation observed to be emitted must work its way through the star, and if there were too much obstruction it would blow up the star." It was realized already in the 20 s that radiation pressure may produce mass loss. However it is only when, in the late $60 \mathrm{~s}$, sensitive UV diagnostics of mass loss from O-star became available that the effects of mass loss on the evolution of stars were really considered.

Radiation triggers mass loss through the line opacities in hot stars. It may also power strong mass loss through the continuum opacity when the star is near the Eddington limit. For cool stars, radiation pressure is exerted on the dust.

For hot stars, typical values for the terminal wind velocity, $v_{\infty}$ is of the order of 3 times the escape velocity, i.e. about $2000-3000 \mathrm{~km} \mathrm{~s}^{-1}$, mass loss rates are between $10^{-8}-10^{-4} \mathrm{M}_{\odot}$ per year. Luminous Blue Variable (LBV) stars show during outbursts mass loss rates as high as $10^{-1}-1 \mathrm{M}_{\odot}$ per year!

According to the recent mass loss rates, $\dot{M}$ behaves with luminosity $L$ like $\dot{M} \sim L^{1.7}$. With the mass-luminosity relation for massive stars, $L \sim M^{2}$, this gives $\dot{M} \sim M^{3.4}$. From this we may estimate the typical timescale for mass loss: $t_{\dot{M}} \sim \frac{M}{M} \sim \frac{1}{M^{2.4}}$. This is to be compared to the MS lifetime $t_{\mathrm{MS}}$. For massive stars, it scales like $t_{\mathrm{MS}} \sim M^{-0.6}$ which shows that with increasing mass the timescale for mass loss decreases much faster than the MS lifetime. One can also estimate the behavior of the amount $\Delta M$ of mass lost with stellar mass $\Delta M \sim M^{2.8} \rightarrow \frac{\Delta M}{M} \sim M^{1.8}$. Thus, not only the amount of mass lost grows with the stellar mass, but even the relative amount of mass loss grows fast with stellar mass, which illustrates the importance of this effect.

In addition to the intensity of the stellar winds for different evolutionary phases, one needs to know how the winds vary with the metallicity. This is a key effect to understand the different massive star populations observed in regions of different metallicities. This has also an important impact on the yields expected from stellar models at various metallicities (see Sect. 6).

Current wisdom considers that very metal-poor stars lose no or very small amounts of mass through radiatively driven stellar winds. This comes from the fact that when the metallicity is low, the number of absorbing lines is small and thus the coupling between the radiative forces and the matter is weak. Wind models impose a scaling relation of the type

$$
\dot{M}(Z)=\left(\frac{Z}{Z_{\odot}}\right)^{\alpha} \dot{M}\left(Z_{\odot}\right)
$$

where $\dot{M}(Z)$ is the mass loss rate when the metallicity is equal to $Z$ and $\dot{M}\left(Z_{\odot}\right)$ is the mass loss rate for the solar metallicity, $Z$ being the mass fraction 
of heavy elements. In the metallicity range from $1 / 30$ to 3.0 times solar, the value of $\alpha$ is between 0.5 and 0.8 according to stellar wind models (Kudritzki et al. 1987; Leitherer et al. 1992; Vink et al. 2001). Such a scaling law implies for instance that a non-rotating $60 \mathrm{M}_{\odot}$ with $Z=0.02$ ends its stellar life with a final mass of $14.6 \mathrm{M}_{\odot}$, the same model with a metallicity of $Z=0.00001$ ends its lifetime with a mass of $59.57 \mathrm{M}_{\odot}$ (cf. models of Meynet \& Maeder 2005 and Meynet et al. 2006 with $\alpha=0.5$ ).

Thus one expects that the metal-poor $60 \mathrm{M}_{\odot}$ star will give birth to a black hole. In that case nearly all (if not all) the stellar mass may disappear in the remnant preventing the star from enriching in substantial way the interstellar medium in new synthesized elements. The metal-rich model on the other hand will probably leave a neutron star and contribute to the enrichment of the ISM through both the winds and the supernova ejecta. When the effects of rotation are accounted for, things can be very different at low metallicity. Mass loss can be boosted due to rotational effects (see Sect. 2.3, 2.4 and 4).

\subsection{Rotational mixing at different $Z$}

Many of the observed characteristics of massive stars require in order to be explained some extra-mixing mechanism working in their radiative zones (see e.g. the review by Maeder \& Meynet 2000a). Rotation appears as the most promising mechanism for explaining these observed characteristics since massive stars are fast rotators and since many instabilities are triggered by rotation (Talon 2008). These instabilities transport angular momentum and chemical species in stellar interiors. Assuming that the star rapidly settles into a state of shellular rotation (constant angular velocity at the surface of isobars), the transport equations due to meridional currents and shear instabilities can be consistently obtained (Zahn 1992). Since the work by J.-P. Zahn, various improvements have been brought to the formulas giving the velocity of the meridional currents (Maeder \& Zahn 1998), those of the various diffusive coefficients describing the effects of shear turbulence (Maeder 1997; Talon \& Zahn 1997; Maeder 2003; Mathis et al. 2004), as well as the effects of rotation on the mass loss (Owocki et al. 1996; Maeder 1999; Maeder \& Meynet 2000b).

Let us recall a few basic results obtained from rotating stellar models (without dynamo process):

1) Angular momentum is mainly transported by the meridional currents. In the outer part of the radiative envelope these meridional currents transport angular momentum outwards. During the Main-Sequence phase, the core contracts and the envelope expands. The meridional currents impose some coupling between the two, slowing down the core and accelerating the outer layers. In the outer layers, the velocity of these currents becomes smaller when the density gets higher, i.e., for a given initial mass, when the metallicity is lower.

2) The chemical species are mainly transported by shear turbulence ${ }^{2}$. Dur-

\footnotetext{
${ }^{2}$ at least in absence of magnetic fields; when magnetic fields are amplified by differential
} 

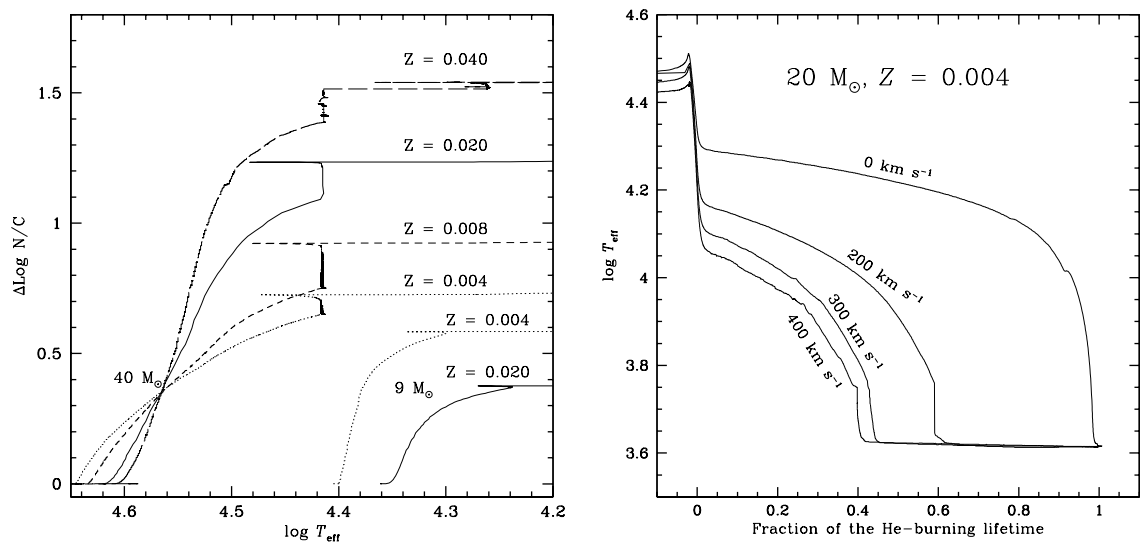

Figure 2: Left panel: Evolution during the MS phase of the N/C ratios (in number) at the surface of rotating stellar models as a function of the effective temperature. The differences in $\mathrm{N} / \mathrm{C}$ ratios are given with respect to the initial values. Figure taken from Meynet \& Maeder (2005). Right panel: Evolution of the $T_{\text {eff }}$ as a function of the fraction of the lifetime spent in the He-burning phase for $20 \mathrm{M}_{\odot}$ stars with different initial velocities. Figure taken from Maeder \& Meynet (2001).

ing the Main-Sequence this process is responsible for the nitrogen enhancements observed at the surface of most OB stars (Maeder et al. 2008). The shear turbulence is stronger when the gradients of the angular velocity are stronger. Due to point 1 above, the gradients of $\Omega$ are stronger in metalpoor stars and thus the mixing of the chemical elements is stronger in these stars. This is illustrated on the left panel of Fig. 2 (see the tracks for the 9 $\mathrm{M}_{\odot}$ stellar models). Looking at the $40 \mathrm{M}_{\odot}$ stellar models, one sees that the higher metallicity model presents the highest surface enrichments, in striking contrast with the behavior of the $9 \mathrm{M}_{\odot}$ model. This comes from the fact that the changes occurring at the surface of the $40 \mathrm{M}_{\odot}$ are not only due to rotation but also to mass loss which is more efficient at higher $Z$ (see also Sect. 6). Mixing is not only more efficient at low metallicity, it is also stronger in stars with increasing initial masses and increasing initial velocities.

The efficiency of the mixing will vary from one element to another. If an element is strongly and rapidly built up in the convective core, it will diffuse by rotational mixing more rapidly in the radiative envelope than an element with a smoother gradient between the convective core and the radiative envelope. This explains why the stellar surface will be more rapidly enriched in nitrogen than in helium.

Some observations indicate that rotational mixing might be more efficient

rotation as in the Tayler-Spruit dynamo mechanism, see Spruit (2002), the main transport mechanism is meridional circulation, see Maeder \& Meynet (2005). 
at lower metallicities (Venn 1999; Venn \& Przybilla 2003) confirming the trend expected from theoretical models. However some variations in the initial distribution of the $\mathrm{CNO}$ elements might somewhat blurr the picture. According to Hunter et al. (2007) the $\mathrm{C} / \mathrm{N}$ and $\mathrm{O} / \mathrm{N}$ ratios in the Small Magellanic Clouds are equal to 7 and 35 respectively, while in the solar neighborhood it is of the order of 4 and 7 (ratios in number). That means that the maximum nitrogen enhancement (obtained at CNO equilibrium) expressed as $\Delta \log (\mathrm{N} / \mathrm{H})$ would be about 1.7 in the SMC and about 1.1 in the solar neighborhood. Thus this effect alone may produce higher nitrogen enrichment in the Magellanic Clouds than in the Galaxy!

In addition to these internal transport processes, rotation also modifies the physical properties of the stellar surface. Indeed the shape of the star is deformed by rotation (a fact which is now put in evidence observationally thanks to the interferometry, see Domiciano de Souza et al. 2003). Rotation implies also a non-uniform brightness (see observational evidences in e.g. Domiciano de Souza et al. 2005). The polar regions are brighter than the equatorial ones. This is a consequence of the hydrostatic and radiative equilibrium (von Zeipel theorem 1924). In addition, as a result of the internal transport processes, the surface velocity and the surface chemical composition are modified.

\subsection{Rotationally induced mass loss at different $Z$}

We can classify the effects of rotation on mass loss in three categories.

1. The structural effects of rotation.

2. The changes brought by rotation on the radiation driven stellar winds.

3. The mechanical mass loss induced by rotation at the critical limit (i.e. when the surface velocity at the equator is such that the centrifugal acceleration balances the gravity).

Let us consider in turn these various processes.

\section{$\underline{\text { Structural effects of rotation on mass loss }}$}

Rotation, by changing the chemical structure of the star, modifies its evolution. For instance, moderate rotation at metallicities of the Small Magellanic Cloud (SMC) favors redward evolution in the Hertzsprung-Russel diagram. This behavior is illustrated in the right panel of Fig. 2 and can account for the high number of red supergiants observed in the SMC (Maeder \& Meynet 2001), an observational fact which is not at all reproduced by non-rotating stellar models.

Now it is well known that the mass loss rates are greater when the star evolves into the red part of the HR diagram, thus in this case, rotation modifies the mass loss indirectly, by changing the evolutionary tracks. The 20 $\mathrm{M}_{\odot}, v_{\text {ini }}=0,200,300$ and $400 \mathrm{~km} \mathrm{~s}^{-1}$ models lose respectively $0.14,1.40$, 1.71 and $1.93 \mathrm{M}_{\odot}$ during the core He-burning phase (see Table 1 in Maeder \& 
Meynet 2001). The enhancement of the mass lost reflects the longer lifetimes of the red supergiant phase when velocity increases. Note that these numbers were obtained assuming that the scaling law between mass loss and metallicity deduced from stellar wind models for hot stars applies during the red supergiant phase. If, during this phase, mass loss comes from continuum-opacity driven wind then the mass-loss rate will not depend on metallicity (see the review by van Loon 2006). In that case, the redward evolution favored by rotation would have a greater impact on mass loss than that shown by these computations.

At very high rotation, the star will have a homogeneous evolution and will never become a red supergiant (Maeder 1987). In this case, the mass loss will be reduced, although this effect will be somewhat compensated by two other processes: first by the fact that the Main-Sequence lifetime will last longer and, second, by the fact that the star will enter the Wolf-Rayet phase (a phase with high mass loss rates) at an earlier stage of its evolution.

\section{$\underline{\text { Radiation driven stellar winds with rotation }}$}

The effects of rotation on the radiation driven stellar winds result from the changes brought by rotation to the stellar surface. They induce changes of the morphologies of the stellar winds and increase their intensities.

\section{Stellar wind anisotropies}

Naively we would first guess that a rotating star would lose mass preferentially from the equator, where the effective gravity (gravity decreased by the effect of the centrifugal force) is lower. This is probably true when the star reaches the critical limit (i.e. when the equatorial surface velocity is such that the centrifugal acceleration exactly compensates the gravity), but this is not correct when the star is not at the critical limit. Indeed as recalled above, a rotating star has a non uniform surface brightness, and the polar regions are those which have the most powerful radiative flux. Thus one expects that the star will lose mass preferentially along the rotational axis. This is correct for hot stars, for which the dominant source of opacity is electron scattering. In that case the opacity only depends on the mass fraction of hydrogen and does not depend on other physical quantities such as temperature. Thus rotation induces anisotropies of the winds (Maeder \& Desjacques 2001; Dwarkadas \& Owocki 2002). Amplitude of the effect for a fast rotating $35 \mathrm{M}_{\odot}$ stellar model can be seen in Fig. 3.

Wind anisotropies have consequences for the angular momentum that a star retains in its interior. Indeed, when mass is lost preferentially along the polar axis, less angular momentum is lost. This process allows loss of mass without too much loss of angular momentum, a process which might be important in the context of the evolutionary scenarios leading to Gamma Ray Bursts (GRB). Indeed in the framework of the collapsar scenario (Woosley 1993), one has to accommodate two contradictory requirements: on one side, the progenitor needs to lose mass in order to have its $\mathrm{H}$ and He-rich envelope 


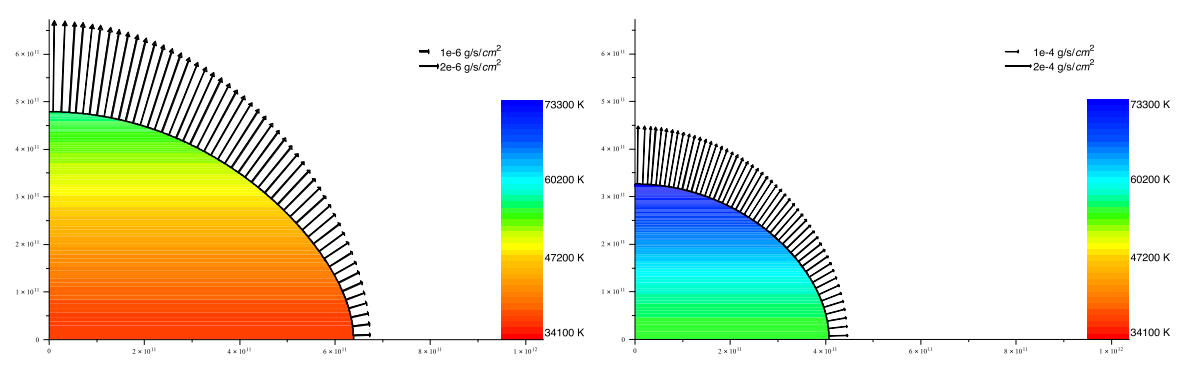

Figure 3: Left panel: Variation of the mass flux at the surface of an initial $35 \mathrm{M}_{\odot}$ at a stage during the core $\mathrm{H}$-burning phase when the mass fraction of hydrogen at the centre is $X_{c}=0.42$. The velocity of the star on the ZAMS is $550 \mathrm{~km} \mathrm{~s}^{-1}$ corresponding to $\Omega / \Omega_{\text {crit }}=0.84$. The star follows a homogeneous evolution. At the stage represented $\Omega / \Omega_{\text {crit }} \sim 1$. Right panel: Same as the left panel, but for a later stage with $X_{c}=0.02$ and $\Omega / \Omega_{\text {crit }} \sim 1$.

removed at the time of its explosion ${ }^{3}$, and on the other hand it must have retained sufficient angular momentum in its central region to give birth to a fast rotating black-hole. Wind anisotropies may help a fast rotating star to lose mass without losing too much angular momentum.

\section{Intensities of the stellar winds}

The quantity of mass lost through radiatively driven stellar winds is enhanced by rotation. This enhancement can occur through two channels: by reducing the effective gravity at the surface of the star, by increasing the opacity of the outer layers through surface metallicity enhancements due to rotational mixing.

- reduction of the effective gravity: The ratio of the mass loss rate of a star with a surface angular velocity $\Omega$ to that of a non-rotating star, of the same initial mass, metallicity and lying at the same position in the HR diagram is given by (Maeder \& Meynet 2000b)

$$
\frac{\dot{M}(\Omega)}{\dot{M}(0)} \simeq \frac{(1-\Gamma)^{\frac{1}{\alpha}-1}}{\left[1-\frac{4}{9}\left(\frac{v}{v_{\text {crit }, 1}}\right)^{2}-\Gamma\right]^{\frac{1}{\alpha}-1}},
$$

where $\Gamma$ is the electron scattering opacity for a non-rotating star with the same mass and luminosity, $\alpha$ is a force multiplier (Lamers et al. 1995). The enhancement factor remains modest for stars with luminosity sufficiently far away from the Eddington limit (Maeder \& Meynet 2000b). Typically, $\frac{\dot{M}(\Omega)}{\dot{M}(0)} \simeq 1.5$ for main-sequence B-stars. In that

\footnotetext{
${ }^{3}$ In case the H-rich envelope would be still present at the time of the explosion, the jets and the gamma photons produced in it would remain invisible for an observer.
} 
case, when the surface velocity approaches the critical limit, the effective gravity decreases and the radiative flux also decreases. Thus the matter becomes less bound when, at the same time, the radiative forces become also weaker. When the stellar luminosity approaches the Eddington limit, the mass loss increases can be much greater, reaching orders of magnitude. This comes from the fact that rotation lowers the maximum luminosity or the Eddington luminosity of a star. Thus it may happen that for a velocity still far from the classical critical limit, the rotationally decreased maximum luminosity becomes equal to the actual luminosity of the star. In that case, strong mass loss ensues and the star is said to have reached the $\Omega \Gamma$ limit (Maeder \& Meynet 2000b).

- Effects due to rotational mixing: During the core helium burning phase, at low metallicity, the surface may be strongly enriched in both $\mathrm{H}$ burning and He-burning products, i.e. mainly in nitrogen, carbon and oxygen. Nitrogen is produced by transformation of the carbon and oxygen produced in the He-burning core and which have diffused by rotational mixing in the H-burning shell (Meynet \& Maeder 2002ab). Part of the carbon and oxygen produced in the He-core also diffuses up to the surface. Thus at the surface, one obtains very high value of the CNO elements, the opacity of the surface increases and thus line driven winds become stronger (see Sect. 4 for quantitative effects).

Mechanical winds induced by rotation

As recalled above, during the Main-Sequence phase the core contracts and the envelope expands. In case of local conservation of the angular momentum, the core would thus spin faster and faster while the envelope would slow down. In that case, it can be easily shown that the surface velocity would evolve away from the critical velocity (see e.g. Meynet \& Maeder 2006). In models with shellular rotation however an important coupling between the core and the envelope is established through the action of the meridional currents. As a net result, angular momentum is brought from the inner regions to the outer ones. Thus, would the star lose no mass by radiation driven stellar winds (as is the case at low Z), one expects that the surface velocity would increase with time and would approach the critical limit. In contrast, when radiation driven stellar winds are important, the timescale for removing mass and angular momentum at the surface is shorter than the timescale for accelerating the outer layers by the above process and the surface velocity decreases as a function of time. It evolves away from the critical limit. Thus, an interesting situation occurs: when the star loses little mass by radiation driven stellar winds, it has more chance to lose mass by a mechanical wind. On the other hand, when the star loses mass at a high rate by radiation driven mass loss then it has no chance to reach the critical limit and thus to undergo a mechanical mass loss. 


\subsection{Spinstars at low metallicity?}

From the previous discussion, it does appear that for a given initial rotation, the effects of rotation will be the more pronounced at low metallicity and this for two reasons:

- Rotational mixing is more efficient at low $Z$.

- Stars more easily evolves toward the critical limit.

Of course if at low metallicity, for whatever reasons, the distribution of the initial velocities is biased toward slow rotators, then the above effects will not be important. Let us however stress here that at present, there is some observational support to the view that the distribution of the initial velocities contains more fast rotators at low Z! Observationally Martayan et al (2006) find that, for B and Be stars, the lower the metallicity, the higher the rotational velocities. The Be stars are stars surrounded by an expanding equatorial disks probably produced by the concomitant effects of both fast rotation and pulsation. Martayan et al. $(2006 ; 2007)$ obtain that Be stars rotate faster than B stars whatever the metallicity is. Thus a high fraction of Be stars reflect a higher fraction of fast rotators. Maeder et al. (1999) and Wisniewski \& Bjorkman (2006) find that the fraction of Be stars with respect to the total number of B and Be stars in clusters with $\log \mathrm{t}(\mathrm{yrs})$ from 7.0 to 7.4 increases when the metallicity decreases. This fraction passes from about $10 \%$ at solar metallicity to about $35 \%$ at the SMC metallicity.

Thus not only for a given initial velocity, theory predicts that the effects of rotation are more pronounced in metal poor regions but also there is some observational hints that there are more fast rotators at low $Z$. Of course we might argue here that the higher proportion of fast rotators results not from a difference in the initial distribution of the velocities but is due to an evolutionary effect. Stars at low $Z$ rotate faster because they lose less angular momentum through radiatively driven stellar winds. However for Btype stars, stellar winds are weak during the Main-Sequence phase and this argument probably does not apply.

From this we conclude that rotation probably has the strongest impact at low metallicity. If we define "spinstars" as stars whose evolution is strongly affected by rotation, then their number should be greater at low $Z$.

Interestingly many observations presently challenge our understanding of the evolution of stars at low and very low metallicity. Let us briefly mention a few of them here: 1) no sign of Pair Instability Supernovae has been observed (Cayrel et al. 2004), 2) a high plateau of the $\mathrm{N} / \mathrm{O}$ ratios (as a function of $\mathrm{O} / \mathrm{H}$ ) are obtained requiring the activity of efficient sources of primary nitrogen (see e.g. Spite et al. 2005), 3) simultaneously the $\mathrm{C} / \mathrm{O}$ ratio as a function of $\mathrm{O} / \mathrm{H}$ shows an upturn at a metallicity of $[\mathrm{O} / \mathrm{H}] \sim-2$ (Cayrel et al. 2004), similar trends are seen in Damped Lyman Alpha systems (Pettini et al. 2008) 4) a significant fraction of very metal poor stars are C-rich stars showing very peculiar abundance pattern at their surface (see the review by Beers \& Christlieb 2005), 5) very helium-rich stars and 6) stars with high 
abundance of sodium and low abundance of oxygen are detected in globular clusters (Gratton et al. 2004; Piotto et al. 2005). As we shall see below rotation may help in understanding these puzzling facts.

\section{The first stellar generations in the Universe}

Understanding the evolution of massive stars at low and very low metallicity is a requirement to address questions such as the nature of the sources of the reionization in the early Universe, the evolution of the interstellar abundances during the early phases of the evolution of galaxies, for finding possible signatures of primordial stellar populations in the integrated light of very distant galaxies and for discovering which objects are the progenitors of the long soft Gamma Ray Bursts. At present, the most "iron" poor objects known in the Universe are not very far from us since they are galactic halo field stars. These objects offer a unique opportunity to study the yields of the first generations of $\operatorname{stars}^{4}$.

In this section, we focus the discussion on the first stars which were made up of material having been processed only by primordial nucleosynthesis, that means from matter essentially deprived of heavy elements. In an environment with primordial composition, one expects the following differences with respect to the more classical evolution at higher metallicities:

- At strictly $Z=0$, the cooling processes, so important for allowing the evacuation of the energy produced when the molecular clouds collapse and thus its fragmentation, are not so efficient as at larger metallicity. This favors the formation of more massive stars. The initial mass function is probably different depending on the mass of the "minihaloes" (see Greif \& Bromm 2006). In minihaloes with masses between $10^{6}$ and $10^{8}$ $\mathrm{M}_{\odot}$, virial temperature is between $10^{3}$ and $10^{4} \mathrm{~K}$ and the cooling is due mainly to the molecules $\mathrm{H}_{2}$. This allows the formation of stars with characteristic masses $\geq 100 \mathrm{M}_{\odot}$. In minihaloes where ionization occurs prior to the late stages of the protostellar accretion process, namely those with a virial temperature superior to $10^{4} \mathrm{~K}$ and thus with masses above $10^{8} \mathrm{M}_{\odot}{ }^{5}$, the hydrogen deuteride (HD) molecule provides an additional cooling channel. In those minihaloes, metal-free gas can cool more efficiently. This leads to the formation of stars with masses superior to $10 \mathrm{M}_{\odot}$. Thus at the very beginning, we would have first, during a quite short period, only very massive Pop III stars and, when gas assembles in more massive haloes (or is reionized by the first stars), a second Pop

\footnotetext{
${ }^{4}$ Note that recently Venn \& Lambert (2008) have challenged the view according to which the C-rich extremely metal poor stars are trustworthy very metal poor stars. But this suggestion, if correct, will not challenge the whole concept of the first stellar generations being traced by metal poor stars because many of the chemical signatures also come from normal stars (i.e. non C-rich stars).

${ }^{5}$ This may also happen in relic HII regions left by the first stars, see Johnson \& Bromm (2006).
} 
III star generation appears with smaller characteristic masses (about 10 $\mathrm{M}_{\odot}$ and above). This population is called by some authors Pop II.5. When the metallicity becomes higher than $10^{-3.5} \mathrm{Z}_{\odot}$, i.e. for $Z$ above about $510^{-6}$ a normal IMF governs the mass distribution of newly born stars $^{6}$. According to Greif \& Bromm (2006) the very massive Pop III stars only contribute marginally to feed the reservoir of ionizing photons and to the chemical enrichment of the interstellar medium. Much more important are on this respect the less massive Pop III stars born in more massive haloes or in relic HII regions.

- The (nearly) absence of heavy elements implies that massive stars cannot ignite hydrogen-burning through the CNO cycle but through the pp chains. However the energy output extracted from the pp chains is not sufficient to compensate for the high luminosity of these stars. The stars must compensate the deficit of nuclear energy by extracting energy from the gravitational reservoir, i.e. they contract. At a given point however, due to this contraction, the central temperature becomes high enough for activating triple $\alpha$ reactions. Some carbon is then produced. When the mass fraction of carbon is of the order of $10^{-12}$, the $\mathrm{CN}$ cycle becomes the main source of energy and the evolution then proceeds as in a more metal rich massive stars. For stars above about $20 \mathrm{M}_{\odot}$, activation of the $\mathrm{CN}$ cycle intervenes very early during the core H-burning (typically before five percents of the hydrogen at the centre is consumed, see Marigo et al. 2003).

- Pop III are more compact due to the contraction they undergo at the beginning of the core H-burning phase (see above) and to the fact that the opacity of primordial material is smaller than that of metal rich one. Typically a Pop III $20 \mathrm{M}_{\odot}$ star on the ZAMS has a radius reduced by a factor 3.5 with respect to the radius of the corresponding star with $Z=0.020$. Even passing from the very low metallicity $Z=10^{-5}$ to 0 already produces a decrease of the radius by a factor 2 ! Smaller radius favors more efficient mixing in two ways: first, for a given value of the diffusion coefficient, $D$, the mixing timescale decreases with the radius as $\sim R^{2} / D$. Second the gradients of $\Omega$ are steeper (see above). As we shall see however, rotational mixing at $Z=0$ is not so efficient as at low but non zero metallicity. We shall come back on that point in the next section.

Recently Ekström et al. (2008) presented a grid of Pop III stellar models including the effects of rotation. Evolutionary tracks of non-rotating and rotating Pop III stellar models are shown in Fig. 4 (left panel). The models are computed until the end of the core Si-burning, except the $9 \mathrm{M}_{\odot}$ that has developed a degenerate core before carbon ignition and has thus been stopped then, and the $15 \mathrm{M}_{\odot}$ model that has been stopped at the end of O-burning

\footnotetext{
${ }^{6}$ Some authors argue that star formation switches to more classical mode already when $Z=10^{-6}$ due to dust production in the early Universe, see Schneider et al. 2006.
} 

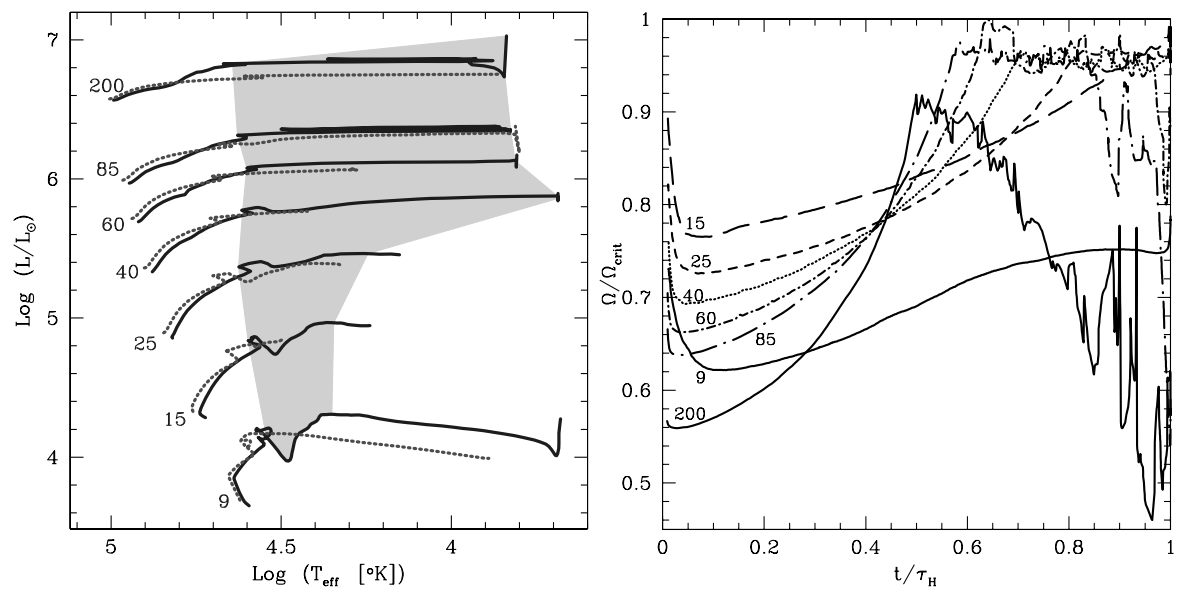

Figure 4: Left panels: Evolution of $Z=0$ models (with rotation: continuous lines; without rotation: dotted lines) in the Hertzsprung Russell diagram. The gray area shows the zone of the diagram where He burns in the core of the rotating models. Right panel: Evolution of the $\Omega / \Omega_{\text {crit }}$ ratio during the MS. All the models start the MS with $v_{\text {eq }}=800 \mathrm{~km} \mathrm{~s}^{-1}$, except the $9 \mathrm{M}_{\odot}$ which starts the MS with $500 \mathrm{~km} \mathrm{~s}^{-1}$. Figures taken from Ekström et al. (2008a).

also because of a too degenerate core at that time. We chose an initial velocity of $800 \mathrm{~km} \mathrm{~s}^{-1}$ on the ZAMS. For the $60 \mathrm{M}_{\odot}$ model, this corresponds to a value of $v / v_{\text {crit }}=0.52$ on the ZAMS, which is slightly superior to 0.4 , the value required at solar metallicity to obtain averaged velocities during the MS phase corresponding to observed values.

We notice that the ZAMS is shifted toward lower effective temperature and luminosity with respect to the non-rotating case $^{7}$. Then, when the evolution proceeds, the tracks become more luminous, and the main-sequence turn-off is shifted to cooler temperature: the core of the rotating models is refueled by fresh $\mathrm{H}$ brought by the mixing. It thus grows, leading to an enhancement of the luminosity.

The onset of the CNO cycle described above can be seen in the HRD: the tracks evolve toward the blue side of the diagram, until the energy provided by the CNO cycle stops the contraction and bends the tracks back in the usual MS feature. In the rotating $9 \mathrm{M}_{\odot}$, this happens at an age of $12.2 \mathrm{Myr}$ (when the central $\mathrm{H}$ mass fraction is $X_{\mathrm{c}}=0.439$ ) while in the non-rotating one it happens a little earlier, at an age of $10.9 \mathrm{Myr}$ (but at a similar burning stage: $\left.X_{\mathrm{c}}=0.439\right)$. In the case of the non-rotating $15 \mathrm{M}_{\odot}$ model, it happens after merely $1.5 \mathrm{Myr}\left(X_{\mathrm{c}}=0.695\right)$, while it takes $2 \operatorname{Myr}\left(X_{\mathrm{c}}=0.677\right)$ in the case of the rotating one. Let us mention that Marigo et al. (2003) find that

\footnotetext{
${ }^{7}$ We recall that this shift is due to the sustaining effect of rotation: the gravity is counter-balanced both by the gas pressure and the centrifugal force in such a way that the star behaves like a lower mass one.
} 
the mass limit for CNO ignition already on the ZAMS is $20 \mathrm{M}_{\odot}$, our results being consistent with that limit.

After central $\mathrm{H}$ exhaustion, the core He-burning phase (CHeB) starts right away: the core was already hot enough to burn a little He during the MS and does not need to contract much further. This prevents the models to start a redward evolution, so they remain in the blue part of the HRD at the beginning of $\mathrm{CHeB}$. Then, something particular happens to the rotating models: because of rotational mixing, some carbon produced in the core is diffused toward the H-burning shell, allowing a sudden ignition of the CNOcycle in the shell. This boost of the shell leads to a retraction of the convective core and a decrease of the luminosity. At the same time, it transforms the quiet radiative $\mathrm{H}$-burning shell into an active convective one. Some primary nitrogen is produced (see more on that point in the next section).

All the models, except the $9 \mathrm{M}_{\odot}$, reach the critical velocity during the MS phase (see the right panel of Fig. (4). Once at critical limit, all the models remain at the critical limit until the end of the Main-Sequence phase. On the right panel of Fig. 4 , the 85 and especially the $200 \mathrm{M}_{\odot}$ seem to depart from $\Omega / \Omega_{\text {crit }}=1$, but this is due to the limit shown here being only the $\Omega$ limit, where the centrifugal force alone is taken into account to counterbalance the gravity. In the two above models however, the radiative acceleration is strong and the models reach the so-called $\Omega \Gamma$-limit, that is the second root of the equation giving the critical velocity: $\overrightarrow{\text { eff }}[1-\Gamma]=\overrightarrow{0}$ (Maeder \& Meynet $2000 \mathrm{~b})$. The true critical velocity is lowered by the radiative acceleration, and though the $\Omega / \Omega_{\text {crit }}$ ratio plotted becomes lower than 1 , these models are actually at the critical limit and remain at this limit till the end of the MS. The mass which is lost by the mechanical winds amounts only to a few percents of the initial stellar mass and thus does not much affect neither their evolution, nor their nucleosynthetic outputs. Much more mass can be lost by mechanical mass loss (see next subsection) when the effects of magnetic fields are accounted for as prescribed in the Tayler-Spruit dynamo theory (Spruit 2002), or when the metallicity is non-zero.

\subsection{Strong mass loss in Pop III stars?}

According to Heger et al. (2003), the fate of single stars depends on their Hecore mass $\left(M_{\alpha}\right)$ at the end of the evolution. They have shown that at very low metallicity, the stars having $64 M_{\odot}<M_{\alpha}<133 M_{\odot}$ will undergo pairinstability and be entirely disrupted by the subsequent supernova. This mass range in $M_{\alpha}$ has been related to the initial mass the star must have on the main sequence (MS) through standard evolution models: $140 M_{\odot}<M_{\text {ini }}<$ $260 M_{\odot}$. However the link between the masses of the He-core mass and the initial mass can be very different depending on the physics considered. Ekström et al. (2008) showed that a $\mathrm{Z}=0,150 \mathrm{M}_{\odot}$ stellar model, having an initial ratio between the equatorial velocity and the critical one equal to

$v_{\text {ini }} / v_{\text {crit }}=0.56$, and computed accounting for the Tayler-Spruit dynamo mechanism (Spruit 2002) and the effects of wind anisotropy (Maeder 1999) 

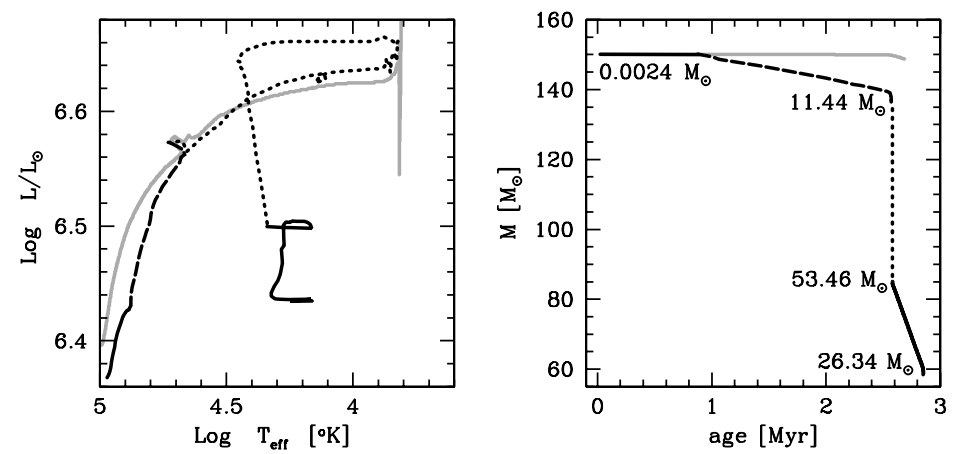

Figure 5: Black line: rotating model; continuous part: beginning of MS $\left(X_{\mathrm{c}}=\right.$ 0.753 down to 0.58 ; dashed part: rest of the MS; dotted part: beginning of core He-burning phase $\left(Y_{\mathrm{c}}=1.00\right.$ down to 0.96$)$; continuous part: rest of the He-burning. Grey line: non-rotating model for comparison. Left panel: evolution in the Hertzsprung-Russell diagram; Right panel: evolution of the mass of the model. The mass indicated is the mass lost at each stage, not a summation. Figures taken from Ekström et al. (2008b).

will lose such great amount of mass that it will reach the end of the core Heburning phase with a mass of $M_{\alpha}$ too small to go through a pair-instability process.

In Fig. 5, we present the evolution in the HR diagram (left panel) and the evolution of mass with time (right panel). The gray line shows a non-rotating model computed with the same physics for comparison. During its whole evolution up to the end of core He-burning, the non-rotating model loses only $1.37 M_{\odot}$. This illustrates the weakness of radiative winds at $Z=0$. The evolution of the rotating model (black line) can be described by four distinct stages:

1. (continuous part, lower left corner in the left panel of Fig. (5) The model starts its evolution on the MS with only radiative winds, losing only a little more than $0.002 M_{\odot}$. During this stage, the ratio of the surface velocity to the critical one increases quickly, mainly because of the strong coupling exerted by the magnetic fields.

2. (dashed part) When its central content of hydrogen is still 0.58 in mass fraction, it reaches the critical velocity and starts losing mass by mechanical mass loss. It remains at the critical limit through the whole MS, but the mechanical wind removes only the most superficial layers that have become unbound, and less than $10 \%$ of the initial mass is lost during that stage $\left(11.44 M_{\odot}\right)$. The model becomes also extremely luminous, and reaches the Eddington limit when $10 \%$ of hydrogen remains in the core. Precisely, it is the so-called $\Omega \Gamma$-limit that is reached here. 
3. (dotted part) The combustion of helium begins as soon as the hydrogen is exhausted in the core, then the radiative H-burning shell undergoes a CNO flash, setting the model on its redward journey. The model remains at the $\Omega \Gamma$-limit and loses a huge amount of mass. The strong magnetic coupling keeps bringing angular momentum to the surface and even the heavy mass loss is not able to let the model evolve away from the critical limit. The mass lost during that stage amounts to $53.46 M_{\odot}$. When the model starts a blue hook in the HR diagram, its surface conditions become those of a WR star $\left(X_{\text {surf }}<0.4\right.$ and $\left.T_{\text {eff }}>10^{\prime} 000 \mathrm{~K}\right)$. The luminosity drops and takes the model away from the $\Gamma$-limit, marking the end of that stage.

4. (continuous part) The rest of the core He-burning is spent in the WR conditions. The mass loss is strong but less than in the previous stage: another $26.34 M_{\odot}$ are lost.

At the end of core He-burning, the final mass of the model is only $M_{\text {fin }}=$ $58 M_{\odot}$, already below the minimum $M_{\alpha}$ needed for PISN $\left(M_{\alpha} \geq 64 M_{\odot}\right)$. Note that the contraction of the core after helium exhaustion brings the model back to critical velocity, so this value for $M_{\text {fin }}$ must be considered as an upper limit.

This result shows that a fast rotating Pop III $150 \mathrm{M}_{\odot}$ may avoid to explode as a PISN. Also such a star will enrich the interstellar medium through its winds. Of course it is by far not certain that the conditions required for such a scenario to occur are met in the first stellar generations but it underlines the fact that fast rotation may drastically change the picture.

Let us note that the nucleosynthetic signature of PISN are not observed in the most metal poor halo stars. Is this due to the above scenario? To the fact that the signature was very quickly erased by the next generations of stars? ${ }^{8}$ Or were such high mass stars not formed? These various hypothesis cannot be disentangled at the present time, but the observation of more and more metal-deficient stars will probably provide elements of response to these questions.

\subsection{Pop III stars as physics laboratories}

The formation and the evolution of the first stellar generations may differ from those of the subsequent generations in many other different ways than the ones quoted above. For instance Pop III stars are supposed to be formed in mini dark haloes. Depending on the nature of dark matter, of its density in the dark halo, annihilation of dark material inside Pop III stars may contribute more or less significantly for compensating their energy losses at the surface.

Recently many authors (see e.g. Spolyar et al. 2008; Iocco et al. 2008; Taoso et al. 2008; Yoon et al. 2008) have studied the effects of dark matter

\footnotetext{
${ }^{8}$ Maybe the metal-poor stars we observe are enriched by more SNe than we actually think, and the later contributions are masking the primordial ones.
} 
annihilation in Pop III stars. In particular Taoso et al. (2008) have obtained that for WIMP densities superior to a critical value (see Fig. 3.2) the MS lifetimes of these models can exceed the present age of the Universe, allowing the existence in the present day Universe of Pop III stars still frozen in their ZAMS evolutionary stage. In contrast to classical ZAMS Pop III stars, these stars would be much more inflated, show lower effective temperatures and surface gravities. The circumstances needed for such stars to exists are probably very rare if ever they have been realised once!

It might be also that fundamental constants had not the same value in the early history of the Universe. The chemical composition of the stellar cores at the end of the He-burning phase depends sensitively on the exact value of the fine structure constant, because variations of the fine structure constant implies changes of the $3 \alpha$ reaction rate. For instance, a variation of a few $10^{-5}$ suffices for instance to avoid any production of oxygen by core He-burning! This deeply affects the advanced evolutionary stages of Pop III massive stars and of course their nucleosynthesis (Ekström et al. in preparation).

\section{Massive star evolution in the low metallicity regime}

At low (but non-zero) metallicities, rotational mixing plays a dominant role and produce two important effects:

First, in this metallicity range, rotating models produce large amounts of primary nitrogen. Although rotational mixing in Pop III stars is by far not a negligible effect, it remains at a relatively modest level due to the absence of strong contraction at the end of the core H-burning phase. On the contrary, when $Z \geq \sim 10^{-10}$, the physical conditions during the core H-burning phase and the core He-burning phase are so different that a strong contraction occurs at the end of the core H-burning phase leading to strong mixing and important primary nitrogen production ${ }^{9}$. For metallicities higher than about 0.001 , rotational mixing is not efficient enough for triggering important primary nitrogen production (at least for the rotational velocities corresponding to the observed ones at this metallicity) and thus rotational mixing, although still important for explaining the surface enrichments does no long change the stellar yields as much as at very low metallicity. Thus primary nitrogen production does appear to go through a maximum when the metallicity decreases from $Z=0.001$ to $Z=0$, supposing that, at the different metallicities, the initial angular momentum content of a given initial mass star on the ZAMS remains more or less constant.

Second, in this metallicity range, rotational mixing, by increasing the CNO surface abundances, might trigger important mass losses through radiatively driven stellar winds. Indeed, another consequence from the strong contraction

\footnotetext{
${ }^{9}$ The presence of a very little amount of metals suffices to boost the efficiency of rotational mixing and the importance of mass loss. In that respect metallicity is like the salt of the cosmos: a small amount is sufficient to enhance its flavor!
} 


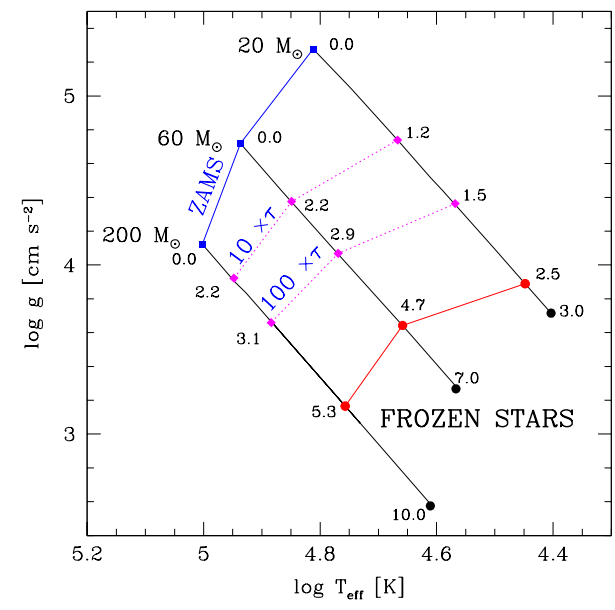

Figure 6: ZAMS positions of 20, 60 and $200 \mathrm{M}_{\odot}$ Pop III stellar models in the surface gravity versus effective temperature plane (both in logarithm) for different dark matter densities (labels in units of $\mathrm{GeV} \mathrm{cm}^{-3}$ ). Big red (gray) circles labeled by 5.3, 4.7 and 2.5, correspond to dark matter densities such that the energy produced by WIMPS annihilation can compensate for the energy losses at the surface. Provided such densities can be sustained for more tan a Hubble time, the star do no long evolve and remains frozen at its ZAMS position. The lines labeled as $10 \tau$ and $100 \tau$ correspond to ZAMS positions of models with a lifetime prolonged by 10 and 100 times with respect to the case without WIMPS. We have adopted a WIMP model with a mass equal to 100 $\mathrm{GeV}$ and a spin-dependent WIMP scattering cross section $\sigma_{\mathrm{SD}}=10^{-38} \mathrm{~cm}^{2}$. Figure taken from Taoso et al. (2008).

at the end of the core $\mathrm{H}$-burning phase is a rapid evolution to the red in the HR diagram. Due to rotational mixing, the opacity in the outer layers increases with time, making appear an outer convective zone. This convective zone dredges-up at the surface great quantities of CNO elements. In our $60 \mathrm{M}_{\odot}$ stellar model with $Z=10^{-8}$ and $v_{\text {ini }}=800 \mathrm{~km} \mathrm{~s}^{-1}$, the CNO content at the surface amounts to one million times the one the star had at its birth (Meynet et al. 2006). Therefore the global metallicity at the surface becomes equivalent to that of a LMC stars while the star began its life with a metallicity which was about 600000 times lower! If we apply the same rules used at higher metallicity relating the mass loss rate to the global metallicity, we obtain that the star may lose about half of its initial mass due to this effect ${ }^{10}$.

There are at least four striking observational facts which might receive an

\footnotetext{
${ }^{10}$ Note that at the moment it is not possible to know if such a rule would apply in those circumstances, i.e. for typically a $60 \mathrm{M} \odot$ Pop III star with an effective temperature of about $6000 \mathrm{~K}$ and a luminosity $\log L / L_{\odot}=6.1$.
} 


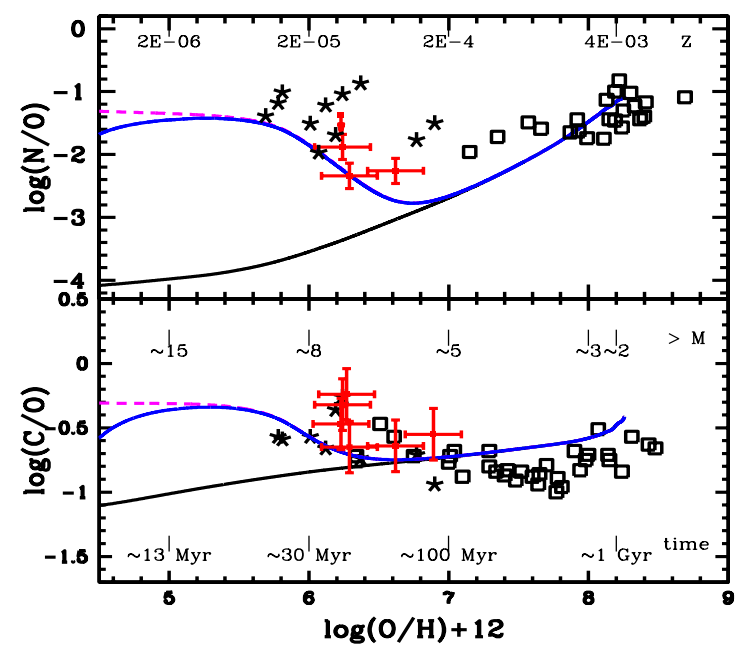

Figure 7: Variation of the $\mathrm{N} / \mathrm{O}$ and $\mathrm{C} / \mathrm{O}$ ratios as a function the $\mathrm{O} / \mathrm{H}$ ratios. $\mathrm{N} / \mathrm{O}$ data points for halo stars are from Israelian et al. (2004, open squares ) and of Spite et al. (2005, stars). C/O points with error bars are for DLA systems from Pettini et al. (2008), C/O data points for halo stars are from Cayrel et al. (2004). The lower continuous curve is the chemical evolution model obtained with the stellar yields of slow rotating $Z=10^{-5}$ models from Meynet \& Maeder (2002) and Hirschi et al. (2004). The dashed line includes the yields of fast rotating $Z=10^{-8}$ models from Hirschi (2007) at very low metallicity. The intermediate curve is obtained using the yields of the $Z=0$ models presented in Ekström et al. (2008) up to $Z=10^{-10}$. The chemical evolution models are from Chiappini et al. (2006a).

explanation based on massive, metal poor, fast rotating stellar models.

Normal metal poor halo stars

The recent observations of the surface abundances of very metal poor halo stars ${ }^{11}$ show the need of a very efficient mechanism for the production of primary nitrogen (Chiappini et al. 2005). As explained in Chiappini et al. (2006a), a very nice way to explain this very efficient primary nitrogen production is to invoke fast rotating massive stars. Very interestingly, fast rotating massive stars help not only in explaining the behavior of the $\mathrm{N} / \mathrm{O}$ ratio at low metallicity but also those of the $\mathrm{C} / \mathrm{O}$. In Fig. 7. predictions for the evolution of $\mathrm{N} / \mathrm{O}$ and $\mathrm{C} / \mathrm{O}$ of chemical evolution models using different sets of yields are compared (Chiappini et al. 2006 ${ }^{12}$ ). We see that the observed

\footnotetext{
${ }^{11}$ These stars are in the field and present $[\mathrm{Fe} / \mathrm{H}]$ as low as -4 , thus well below the metallicities of the globular clusters.

${ }^{12}$ The details of the chemical evolution models can be found in Chiappini et al. (2006b),
} 
$\mathrm{N} / \mathrm{O}$ ratio is much higher than what is predicted by a chemical evolution model using the yields of the slow-rotating $Z=10^{-5}$ models from Meynet \& Maeder (2002) down to $Z=0$. When adding the yields of the fast-rotating $Z=10^{-8}$ models from Hirschi (2007) the fit is much improved. The same improvement is found for the $\mathrm{C} / \mathrm{O}$ ratio, which presents an upturn at low metallicity. Thus these comparisons support fast rotating massive stars as the sources of primary nitrogen in the galactic halo.

High N/O and the $\mathrm{C} / \mathrm{O}$ upturn of the low-metallicity stars are also observed in low-metallicity DLAs (Pettini et al. 2008, see the crosses in Fig. 7). We note that the observed points are below the points for the halo stars in the $\mathrm{N} / \mathrm{O}$ versus $\mathrm{O} / \mathrm{H}$ plane. This may be attributed to two causes: either the observed $\mathrm{N} / \mathrm{O}$ ratios observed in halo stars are somewhat overestimated or the difference is real and might be due to different star formation histories in the halo and in DLAs. Let us just discuss these two possibilities.

Measures of nitrogen abundances at the surface of very metal poor stars is quite challenging, much more than the measure of nitrogen in the interstellar medium as is done for the DLAs, therefore one expects that the data for DLAs suffer much smaller uncertainties than those for halo stars. In that respect the observed $\mathrm{N} / \mathrm{O}$ ratios in DLAs give more accurate abundances than halo stars. Most probably the star formation history in DLAs is not the same as in the halo. While, as recalled above, in the halo we see the result of a strong and rapid star formation episode, in DLAs one might see the result of much slower and weaker star formation episodes. In that case, both massive stars and intermediate mass stars contributed to the build up of the chemical abundances and the chemical evolution models presented in Fig. 7 do no longer apply to these systems (see Chiappini et al. 2003; Dessauges-Zavadsky 2007 for chemical evolution models of DLAs). It will be very interesting to study the results of chemical evolution models adapted to this situation and accounting for stellar yields from both rotating massive and intermediate stars. Let us just mention at this stage that primary nitrogen production in metal poor intermediate mass stars is also strongly favored when rotational mixing is accounted for (Meynet \& Maeder 2002). Thus also in that case, rotation may play a key role.

The primary nitrogen production is accompanied by other interesting features such as the production of primary ${ }^{13} \mathrm{C}$ (see Chiappini et al. 2008). Production of primary ${ }^{13} \mathrm{C}$ by massive stars can explain the low ${ }^{12} \mathrm{C} /{ }^{13} \mathrm{C}$ ratios found recently by Spite et al. (2006) for normal very metal poor halo stars without invoking the contribution of AGBs which, according to the chemical evolution models for the halo, did not play a major role bellow $[\mathrm{Fe} / \mathrm{H}] \sim-2.5$. This is important as recent observational results (Melendez \& Cohen 2007) found the ${ }^{25} \mathrm{Mg} /{ }^{24} \mathrm{Mg},{ }^{26} \mathrm{Mg} /{ }^{24} \mathrm{Mg}$ ratios in halo metal poor stars to be low, again suggesting that AGB stars would have played a minor role below $[\mathrm{Fe} / \mathrm{H}]$ $\sim-2.0$. The primary nitrogen production is also accompanied by the produc-

where they show that such a model reproduces nicely the metallicity distribution of the Galactic halo. This means that the timescale for the enrichment of the medium is well fitted. 
tion of primary ${ }^{22} \mathrm{Ne}$. Primary ${ }^{22} \mathrm{Ne}$ is produced by diffusion of primary nitrogen from the H-burning shell to the core He-burning zone, or by the engulfment of part of the H-burning shell by the growing He-burning core. These processes occur in rotating massive star models (Meynet \& Maeder 2002; Hirschi 2007). In the He-burning zone, ${ }^{14} \mathrm{~N}$ is transformed into ${ }^{22} \mathrm{Ne}$ through the classical reaction chain ${ }^{14} \mathrm{~N}(\alpha, \gamma){ }^{18} \mathrm{~F}\left(\beta^{+} \nu\right){ }^{18} \mathrm{O}(\alpha, \gamma){ }^{22} \mathrm{Ne}$.

In the He-burning zones (either in the core at the end of the core $\mathrm{He}-$ burning phase or in the He-burning shell during the core C-burning phase and in the following convective C-burning shell), neutrons are released through the reaction ${ }^{22} \mathrm{Ne}(\alpha, \mathrm{n})^{25} \mathrm{Mg}$. These neutrons then can either be captured by iron seeds and produce s-process elements or be captured by light neutron poisons and thus be removed from the flux of neutrons. The final outputs of s-process elements will depend on at least three factors: the amounts of 1.${ }^{22} \mathrm{Ne}, 2$.- neutron poisons and 3.- iron seeds. In standard models (without rotation), when the metallicity decreases, the amount of ${ }^{22} \mathrm{Ne}$ decreases (less neutrons produced), the strength of primary neutron poisons becomes relatively more important in particular for $[\mathrm{Fe} / \mathrm{H}] \leq-2$ with respect to solar, and the amount of iron seeds also decreases (e.g., Raiteri et al. 1992). Thus very small quantities of s-process elements are expected. When primary nitrogen and therefore primary ${ }^{22} \mathrm{Ne}$ is present in quantities as given by rotating models which can reproduce the observed trends for the $\mathrm{N} / \mathrm{O}$ and $\mathrm{C} / \mathrm{O}$ ratios in the halo stars, then a very different output is obtained (Pignatari et al. 2008). The abundances of several s-process elements are increased by many orders of magnitudes. In particular, the elements are produced in the greatest quantities in the atomic mass region between strontium and barium, and no long in the atomic mass region between iron and strontium as in the case of standard models.

These first results show that some heavy s-process elements, not produced in standard models (without rotation), might be produced in significant quantities in metal poor rotating stellar models. It will be very interesting in the future to find some non ambiguous signature of the occurrence of this process in the abundance pattern of very metal poor halo stars.

C-rich Extremely Metal Poor Stars (CEMP)

Below about $[\mathrm{Fe} / \mathrm{H}]<-2.5$, a significant fraction of very iron-poor stars are C-rich (see the review by Beers \& Christlieb (2005). Some of these stars show no evidence of $s$-process enrichments by AGB stars and are thus likely formed from the ejecta of massive stars. The problem is how to explain the very high abundances with respect to iron of CNO elements. As shown by Meynet et al. (2006) and Hirschi (2007) the matter released in the winds of fast rotating very metal poor massive stars is enriched in both $\mathrm{H}$ - and He-burning products and present striking similarities with the abundance patterns observed at the surface of CEMPs and these authors proposed that at least some of these stars might be formed from the winds of such objects. Note that stars formed in that way should also be He-rich! It is likely that rotation also affects the composition of the ejecta of intermediate mass stars. Meynet et al. (2006) 
predict the chemical composition of the envelope of a $7 \mathrm{M}_{\odot}$ E-AGB star which have been enriched by rotational mixing. The composition also presents striking similarities with the abundance patterns observed at the surface of CEMPs. The presence of overabundances of fluorine (Schuler et al. 2007) and of $s$-process elements might be used to discriminate between massive and intermediate mass stars.

\section{He-rich stars in globular clusters}

Indirect observations indicate the presence of very helium-rich stars in the globular cluster $\omega$ Cen (Piotto et al. 2005). Stars with a mass fraction of helium, $Y$, equal to 0.4 seem to exist, together with a population of normal helium stars with $Y=0.25$. Other globular clusters appear to host helium-rich stars (Caloi \& D'Antona 2007), thus the case of $\omega$ Cen is the most spectacular but not the only one. There is no way for these very low mass stars to enrich their surface in such large amounts of helium and one possibility is that they have acquired their He abundance from the protostellar cloud from which they formed (an alternative would be through mass transfer in close binary systems). Where does this helium come from? We proposed that it was shed away by the winds of metal poor fast rotating stars (Maeder \& Meynet 2006).

\section{Chemical anomalies in globular clusters}

In globular clusters, stars made of material only enriched in H-burning products have been observed (see the review by Gratton et al. 2004). Probably these stars are also enriched in helium and thus this observation is related to the one reported just above. The difference is that proper abundance studies can be performed for carbon, nitrogen, oxygen, sodium, magnesium, lithium, fluorine ..., while for helium only indirect inferences based on the photometry can be made. Decressin et al. (2007a) propose that the matter from which the stars rich in H-burning products are formed, has been released by slow winds of fast rotating massive stars. Other authors have proposed AGB stars as the main supplier of the material from which the Na-rich and Opoor stars are formed (see e.g. D'Antona \& Ventura 2008). The massive star origin presents however some advantages: first a massive star can induce star formation in its surrounding, thus two effects, the enrichment and the star formation can be triggered by the same cause. Second, the massive star scenario allows to use a less flat IMF than the scenario invoking AGB stars (Prantzos \& Charbonnel 2006). The slope of the IMF might be even a Salpeter's one in case the globular cluster lost a great part of its first generation stars by tidal stripping (Decressin et al. 2007b; Decressin et al. 2008).

All the above observations seem to point toward the same direction, an important population of spinstars at low Z. Of course alternative explanations exist for all these features. One advantage of those presented above is that they rely on one unique physical process: rotational mixing! 


\section{Massive star evolution in the near solar metal- licity regime}

In the near solar metallicity regime, rotation and mass loss by stellar winds are of similar importance. Neither of the two aspects can be neglected. This metallicity range is also the one in which models can be checked and calibrated by comparisons with well observed features either of individual stars or of stellar populations. Among these observed features let us cite

- The observed changes of the surface abundances.

- The observed changes of the surface velocities.

- The shape of fast rotating stars measured by interferometric technics, variation of the effective temperature with the colatitude, measures of wind anisotropies, shape of nebulosities resulting from outbursts.

- The sizes of the convective cores as deduced from asterosismic analysis. Asterosismic observations can also constrain the interior variation of the angular velocity.

- The width of the Main Sequence band.

- The existence and variation with $Z$ of the populations of Be stars.

- The variation with $Z$ of the blue to red supergiant ratio.

- The variation with $Z$ of the Wolf-Rayet populations.

- The rotation rates of young pulsars.

- The variation with $Z$ of different core collapse supernova types.

As a general statement, it does appear that models including the effects of rotation provide a much better fit to most of the above observed features. Here we shall discuss two of them, the cases of surface abundances and the variation with $Z$ of the ratio of type Ibc to type II supernovae.

A rotating star is predicted to present some nitrogen surface enrichment already during the main sequence. The amplitude of the nitrogen enrichment at the surface depends on the initial mass (increases with the mass), the age (increases with the age) and the initial rotational velocity (increase with the initial velocity). Thus we see that the nitrogen surface abundance is at least a function of three parameters: mass, age and velocity. This is correct as long as we consider stars with a given initial composition (rotational mixing is more efficient at low Z) and whose evolution is not affected by a close binary companion.

To see a relation between N-enrichment and velocity, it is necessary to use stars with different rotational velocities but having similar masses and ages. In Fig. 8, such a relation is shown for stars in the N11 SMC cluster 


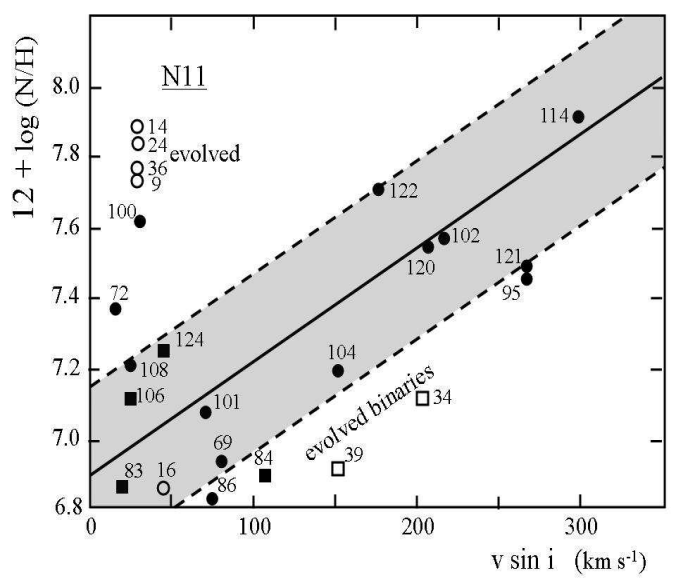

Figure 8: The $\mathrm{N}$ abundance (in a scale where $\log H=12.0$ ) as a function of $v \sin i$ for the MS stars (black dots) in N11 with masses between 14 and $20 \mathrm{M}_{\odot}$ according to Hunter (2008, private communication). The binaries are shown by a square. The evolved stars in a band of $0.1 \mathrm{dex}$ in $\log T_{\text {eff }}$ beyond the end of the MS are shown with open symbols. The gray band indicates uncertainties of \pm 0.25 dex. Figure taken from Maeder et al. (2008).

(Maeder et al. 2008), where the sample is limited to the stars in the mass range 14 to $20 \mathrm{M}_{\odot}$ on the basis of the data provided by Hunter $(2008$, private communication) and in the formal MS band as given by Fig. 34 from Hunter et al. (2007).

We see that the bulk of stars in N11 shows a relation of the excess of N/H depending on $v \sin i$ (the mean square root of the data for the MS band stars is 0.23 dex from the data by Hunter 2008, private communication, the scatter in $v \sin i$ is not given). The amplitude of the $(\mathrm{N} / \mathrm{H})$ is about $0.6 \mathrm{dex}$ for velocities of $200 \mathrm{~km} \mathrm{~s}^{-1}$, slightly higher than the value obtained from rotating stellar models for $Z=0.02$ for the corresponding masses (see Fig. 1 in Maeder et al. 2008).

When data samples, limited in masses and ages, are used, a very nice correlation is found between the surface N-enrichment and $v \sin i$ (see also Fig. 4 in Maeder et al. 2008), supporting a $\mathrm{N}$ enrichment depending on rotational velocities. Stars beyond the end of the MS phase do not obey to such a relation, because their velocities converge toward low values (see Fig. 12 by Meynet \& Maeder 2000). A fraction, which we estimate to be $\sim 20 \%$ of the stars, may escape from the relation as a result of binary evolution, either by tidal mixing or mass transfer.

Core collapse supernovae of type Ib and Ic are very interesting events for many reasons. One of them is that in four cases, the typical spectrum of a type Ic supernova has been observed together with a long soft Gamma Ray Burst 

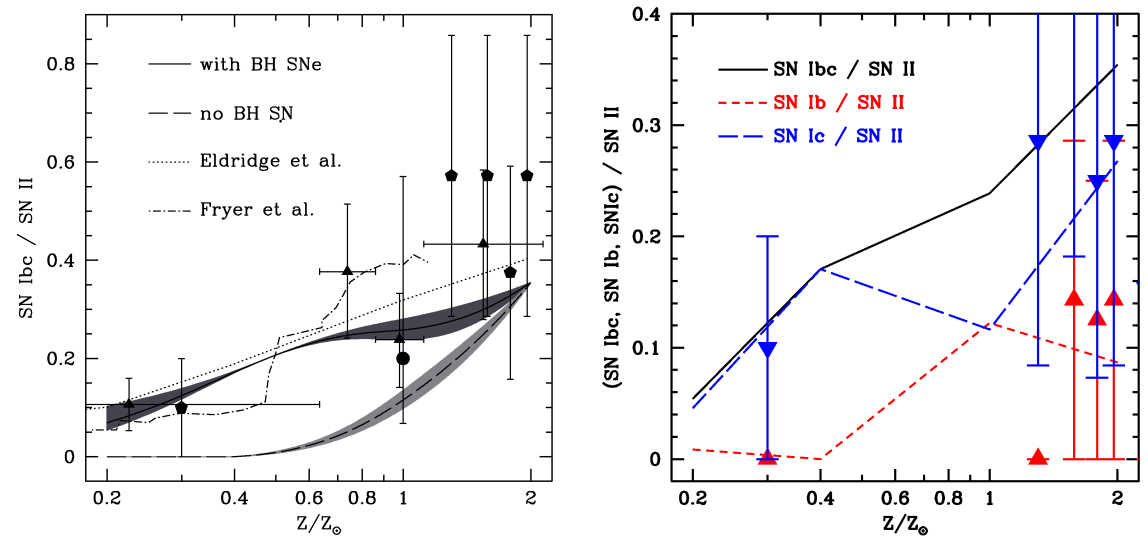

Figure 9: Left panels: Rate of SN Ibc / SN II if all models produce a SN (solid line) or if models producing a black holes do not explode in a SN (dashed line). Grey areas are the corresponding estimated errors from our models. Pentagons are observational data from Prieto et al. (2008), triangles are data from Prantzos \& Boissier (2003) and circle is the measurement at solar metallicity from Cappellaro \& Turatto (2001). The dotted line represents the binary models of Eldridge et al. (2008), and the dotted-dashed line the rate obtained with the binary models of Fryer et al. (2007). Right panel: Rates of SN Ic / SN II (blue long-dashed line), SN Ib / SN II (red short-dashed line) and SN Ibc / SN II (black solid line). The points are extracted from the data of Prieto et al. (2008): triangles (red) represent the observed SN Ib / SN II rate, and upside-down triangles (blue) the observed SN Ic / SN II rate. Each triangle corresponds to a sample of 11 core collapse SNe (color figure available online). Figure taken from Georgy et al. submitted.

(GRB) event (Woosley \& Bloom 2006). Also, recent observations (Prieto et al. 2008) present new values for the variation with the metallicity of the number ratio (SN Ib + SN Ic)/SN II to which theoretical predictions can be compared. Finally, according at least to single star scenarios, these supernovae arise from the most massive stars. They offer thus a unique opportunity to study the final stages of these objects which have a deep impact on the photometric and spectroscopic evolution of galaxies and also contribute to its chemical evolution .

We shall now discuss the predictions of single star models for the type $\mathrm{Ib} / \mathrm{Ic}$ supernovae frequency. Since these supernovae do not show any H-lines in their spectrum, they should have as progenitors stars having removed at least their H-rich envelope by stellar winds, i.e. their progenitors should be WR stars of the WNE type (stars with no $\mathrm{H}$ at their surface and presenting $\mathrm{He}$ and $\mathrm{N}$ lines) or of the WC/WO type (stars with strong overabundances of He-burning products at their surface, mainly carbon and oxygen). 
Considering that all models ending their lifetime as a WNE or WC/WO phase will explode as a type Ibc supernova, it is possible to compute the variation with the metallicity of the number ratio of type Ibc to type II supernovae. The result is shown in Fig. 9 (left panel, see also Meynet \& Maeder 2005). One sees that this ratio increases with the metallicity. This is due to the fact that at higher metallicity, the minimum initial mass of stars ending their life as WNE, or WC/WO stars is lower than at lower metallicities. Single star models can reasonably well reproduce the observed trend with the metallicity. They however give slightly too small values with respect to the observations, which may indicate that a portion of the type Ibc supernovae may originate from close binary evolutions. Models accounting for single and binary channel (but without rotation) are shown as a dotted line (Eldridge et al. 2008). They provide a good fit to the observations. But in that case most of the supernovae originate from the binary channel, leaving little place for the single star scenario. These models would also predict that most of the WR stars are the outcome of close binary evolution. This does not appear to be confirmed by the observations of Foellmi et al (2003ab). Most likely, both the single and binary channel contribute.

The right panel of Fig. 9 shows how the number ratio of type Ib and type Ic supernovae considered separately compare with the observations. We see that a good agreement is obtained although the observations are still scarce and based on a small number of cases.

Of course the situation may change in case, when a Black Hole (BH) is formed, no SN event occurs. We computed new (SN Ib + SN Ic)/ SN II ratios with the assumption that all models massive enough to form a black hole do not produce a SN. Comparing with the observed rates in the left panel of Fig. 9 (see dashed line) we see that in the case no supernova event occurs when a BH is formed, single star models might still account for a significant fraction of the type Ibc supernovae for $Z>0.02$. At $Z=0.004$ all type Ibc should arise from other evolutionary scenarios. A possibility would be in that case to invoke close binary evolution with mass transfer.

Probably, the hypothesis according to which no supernova event is associated when a BH is formed, is too restrictive. For instance, the collapsar scenario for Gamma Ray Bursts (Woosley 1993) needs the formation of a black holes (Dessart, private communication) and this formation is at least accompanied in some cases by a type Ic supernova event. Also the observation of the binary system GRO J1655-40 containing a black hole (Israelian et al. 1999) suggest that a few stellar masses have been ejected and that a SN event occurred when the $\mathrm{BH}$ formed. This is deduced from the important chemical anomalies observed at the surface of the visible companion, chemical anomalies whose origin is attributed to the fact that the (now visible) companion accreted part of the SN ejecta. This gives some support to the view that, at least in some cases, the collapse to a BH does not prevent mass ejection and a supernova event to occur.

As emphasized at the beginning of this section, rotation and mass loss probably are both important in this metallicity range. Impact of these two 
processes on various outputs of solar metallicity stellar models can be found in Heger \& Langer (2000), Meynet \& Maeder (2000; 2003), Hirschi et al. (2005a).

\section{Massive star evolution at metallicities above the solar metallicity}

Above solar metallicities, radiative line driven winds become the dominant factor affecting the evolution of massive stars. In models with moderate rotation, we note however that the effects of rotational mixing are still important but their impact is less apparent being somewhat mixed with those of the stellar winds. From a theoretical point of view we can note two features which do appear different at high metallicity: first the chemical enrichment of the interstellar medium is different. Indeed, as been shown by Maeder (1992), when the stellar winds are important, greater quantities of products from the early phases of the evolution of stars are ejected into the interstellar medium when comparison is made with the chemical abundances of the ejecta of a similar star which would have released its outer layers only at the supernova stage. Physically this comes from the fact that when a layer of stellar material is ejected by stellar winds, it is ejected at an early phase of the evolution of the star, when the layer has only been partially processed by the nuclear reactions. This allows the production of some elements to be enhanced and other to be decreased with respect to a star releasing its outer layers only at the supernova stage. Computations shows that at high metallicity, greater quantities of helium, carbon (Maeder 1992), fluorine (Meynet \& Arnould 2000; Palacios et al. 2005a), aluminum 26 (Palacios et al. 2005b), s-process elements (Arnould et al. 2006) will be ejected. This may have interesting consequences for the chemical evolution of the galaxies at high metallicity.

Another interesting difference which occurs at high metallicity is the evolution of the angular momentum. As we explained in Sec. 2, one expects that at high metallicity, angular momentum is more easily transported from the core to the surface and more easily ejected at the surface by the stellar winds. Thus, everything being equal, one would expect that the angular momentum of the central regions will be lower when the metallicity is higher. Together with the fact that Black-Hole formation is probably more difficult at high $Z$ (also because of the strong stellar winds), this makes the formation of collapsars which are considered as a serious candidate for long soft gamma ray burst much less favorable at high metallicity (see e.g. Hirschi et al. 2005b). In case the rotation rate of young pulsar depends to some extent to the rotation rate of the core at the presupernova stage, the above line of reasoning would lead to the conclusion that the rotation rate of young pulsars should be slower in metal rich regions than in metal poor ones. 


\section{Conclusion}

Massive star evolution is at the crossroad of many topical astrophysical problems: their link with gamma-ray line astrophysics, the origin of galactic cosmic rays, that of isotopic anomalies in the meteorites, the many puzzling observed features related to very metal poor stars in the field of the halo, to the stars in globular clusters, to the progenitors of core collapse supernovae and gamma ray bursts, to neutron stars and black holes, place them at the heart of modern astrophysics. Moreover they represent unique tool to probe the distant universe and constitute important sources of radiations, of new synthetised nuclei and of momentum in galaxies. Still major improvements of massive star models are needed. Great deal of efforts have been made to provide more realistic models accounting for the effects of mass loss and rotation. Aspects as the effects of accretion processes during the pre-main sequence phase, magnetic fields, tidal forces in close binaries, still remain to be further explored and represent wonderful challenges for future works.

\section{References}

Arnould, M., Goriely, S., Meynet, G. 2006, A\&A 453, 653

Beers, T.C., Christlieb, N. 2005, ARAA 43, 531

Caloi, V., D'Antona, F. 2007, A\&A 463, 949

Cappellaro, E., Turatto, M. 2001, The influence of binaries on stellar population studies, Dordrecht: Kluwer Academic Publishers, 2001, xix, 582 p. Astrophysics and space science library (ASSL), Vol. 264. ISBN 0792371046, p.199

Casagrande, L., Flynn, C., Portinari, L., Girardi, L., Jimenez, R. 2007, MNRAS 382,1516

Cayrel, R., Depagne, E., Spite, M. 2004, A\&A 416, 1117

Chiappini, C., Matteucci, F., Meynet, G. 2003, A\&A 410, 257

Chiappini, C., Matteucci, F., Ballero, S. K. 2005, A\&A 437, 429

Chiappini, C., Hirschi, R., Meynet, G., Ekström, S., Maeder, A., Matteucci, F. 2006a, A\&A Letters 449, 27

Chiappini, C., Hirschi, R., Matteucci, F., Meynet, G., Ekström, S., Maeder, A. 2006b, in "Nuclei in the Cosmos IX", Proceedings of Science, 9 pages (arXiv:astro-ph/0609410)

Chiappini, C., Ekström, S.,Hirschi, R., Meynet, G., Maeder, A., Charbonnel, C. 2008, A\&A Letters 479, 9

Cayrel, R., Depagne, E., Spite, M. et al. 2004, A\&A 416, 1117

D’Antona, F., Ventura, P. 2008, The Messenger 134, 18

Decressin, T., Baumgardt, H., Kroupa, P. 2008, A\&A 492, 101

Decressin, T., Charbonnel, C., Meynet, G. 2007b, A\&A 475, 859

Decressin, T., Meynet, G., Charbonnel, C., Prantzos, N., Ekström, S. 2007a, A\&A 464,1029 
Dessauges-Zavadsky, M., Calura, F., Prochaska, J. X., D’Odorico, S., Matteucci, F. 2007, A\&A 470, 431

Domiciano de Souza, A., Kervella, P., Jankov, S., Abe, L., Vakili, F., di Folco, E., Paresce, F. 2003, A\&A 407, L47

Dwarkadas, V. V., Owocki, S. P. 2002, ApJ 581, 1337

Eddington, A.S. 1926, in "The Internal Constitution of The Stars", Cambridge University, Cambridge.

Ekström, S., Meynet, G., Chiappini, C., Hirschi, R., Maeder, A. 2008a, A\&A 489, 685

Ekström, S., Meynet, G., Maeder, A. 2008b, IAU Symposium 250, p. 209

Eldridge, J. J., Izzard, R. G., Tout, C. A. 2008, MNRAS 384, 1109

Foellmi, C., Moffat, A. F. J., Guerrero, M. A. 2003a, MNRAS 338, 360

Foellmi, C., Moffat, A.F. J., Guerrero, M. A. 2003b, MNRAS 338, 1025

Fryer, C. L., Mazzali, P. A., Prochaska, J. et al. 2007, PASP 119, 1211

Gratton, R., Sneden, C., Carretta, E. 2004, ARA\&A 42, 385

Greif, T.H., Bromm, V. 2006, MNRAS 373, 128

Heger, A., Langer, N. 2000, ApJ 544, 1016

Heger, A., Fryer, C. L., Woosley, S. E., Langer, N., Hartmann, D. H. 2003, ApJ 591, 288

Hirschi, R. 2007, A\&A 461, 571

Hirschi, R., Meynet, G., Maeder, A. 2004, A\&A 425, 649

Hirschi, R., Meynet, G., Maeder, A. 2005a, A\&A 433, 1013

Hirschi, R., Meynet, G., Maeder, A. 2005b, A\&A 443, 581

Hunter, I., Dufton, P. L., Smartt, S. J. et al. 2007, ApJ 466, 277

Iocco, F., Bressan, A., Ripamonti, E., Schneider, R., Ferrara, A., Marigo, P. 2008, MNRAS 390, 1655

Israelian, G. et al. 1999, Nature 401, 142

Israelian, G., Ecuvillon, A., Rebolo, R., García-López, R., Bonifacio, P., Molaro, P. 2004, A\&A 421, 649

Johnson,J. L., Bromm, V. 2006, MNRAS 366, 247

Kudritzki, R.P., Pauldrach, A. W. A, Puls, J. 1987, A\&A 173, 293

Lamers, H.J.G.L.M., Snow, T.P., Lindholm, D.M. 1995, ApJ 455, 269

Leitherer, C., Robert, C., Drissen, L. 1992, ApJ 401, 596

Maeder, A. 1987, A\&A 158, 179

Maeder, A. 1992, A\&A 264, 105

Maeder, A. 1997, A\&A 321, 134

Maeder, A. 1999, A\&A 347, 185

Maeder, A. 2003, A\&A 399, 263

Maeder, A., Desjacques, V. 2001, A\&A 372, L9 
Maeder, A., Meynet, G. 2000a, ARA\&A 38, 143

Maeder, A., Meynet, G. 2000b, A\&A 361, 159

Maeder, A., Meynet, G. 2001, A\&A 373, 555 (paper VII)

Maeder, A., Meynet, G. 2005, A\&A 440, 1041

Maeder, A., Meynet, G. 2006, A\&A 448, L37

Maeder, A., Zahn, J.P. 1998, A\&A 334, 1000

Maeder, A., Grebel, E.K., Mermilliod, J.-C. 1999, A\&A 346, 459

Maeder, A., Meynet, G., Ekstrom, S., Georgy, C. 2008, Comm. in Asteroseismology, Contribution to the Proceedings of the 38th LIAC, HELAS-ESTA, BAG, in press (arXiv:0810.0657)

Marigo, P., Chiosi, C., Kudritzki, R.-P. 2003, A\&A 399, 617

Martayan, C., Frémat, Y., Hubert, A.-M. et al. 2006, A\&A 452, 273

Martayan, C., Frémat, Y., Hubert, A.-M., Floquet, M., Zorec, J., Neiner, C. 2007, A\&A 462, 683

Mathis, S., Palacios, A., Zahn, J.-P. 2004, A\&A 425, 243

Matteucci, F. 2001, The chemical evolution of the Galaxy, Astrophysics and space science library, Volume 253, Dordrecht: Kluwer Academic Publishers, ISBN 0-7923-6552-6, 2001, XII + 293 pp.

Melendez, J., Cohen, J.G. 2007, ApJ 659, L25

Meynet, G., Arnould, M. 2000, A\&A 355, 176

Meynet, G.; Maeder, A. 2000, A\&A 361, 101

Meynet, G., Maeder, A., 2002a, A\&A 381, L25

Meynet, G., Maeder, A., 2002b, A\&A 390, 561

Meynet, G., Maeder, A., 2003, A\&A 404, 975

Meynet, G., Maeder, A., 2005, A\&A 429, 581

Meynet, G., Maeder, A. 2006, in Stars with the B[e] Phenomenon. ASP Conf. Ser., eds. M. Kraus \& A.S. Miroshnichenko, 355, p. 27

Meynet, G., Ekstroem, S., Maeder, A. 2006, A\&A 447, 623

Mowlavi, N., Meynet, G., Maeder, A., Schaerer, D., Charbonnel, C. 1998, A\&A 335, 573

Owocki, S.P., Cranmer, S.R., Gayley, K.G. 1996, ApJ 472, L115

Palacios, A., Arnould, M., Meynet, G. 2005a, A\&A 443, 243

Palacios, A., Meynet, G., Vuissoz, C. 2005b, A\&A 429, 613

Pettini, M., Zych, B. J., Steidel, C. C., Chaffee, F. H. 2008, MNRAS 385, 2011

Pignatari, M., Gallino, R., Meynet, G., Hirschi, R., Herwig, F., Wiescher, M. 2008, ApJ 687, L95

Piotto, G., Villanova, S., Bedin, L. R. 2005, ApJ 621, 777

Prantzos, N., Boissier, S. 2003, A\&A 406, 259

Prantzos, N., Charbonnel, C. 2006, A\&A 458, 135 
Prieto, J. L., Stanek, K. Z., Beacom, J. F. 2008, ApJ 673, 999

Raiteri, C. M., Gallino, R., Busso, M. 1992, ApJ 387, 263

Schneider, R., Salvaterra, R., Ferrara, A., Ciardi, B. 2006, MNRAS 369, 825

Schuler, S. C., Cunha, K., Smith, V.V., Sivarani, T., Beers, T. C.; Lee, Y.S. 2007, ApJ 667, L81

Spite, M., Cayrel, R., Plez, B. 2005, A\&A 430, 655

Spite, M., Cayrel, R., Hill, V. et al. 2006, A\&A 455, 291

Spolyar, D., Freese, K., Gondolo, P. 2008, Physical Review Letters 100, 051101

Spruit, H. C. 2002, A\&A 381, 923

Talon, S. 2008, EAS Publications Series 32, 2008, 81

Talon, S., Zahn, J.P. 1997, A\&A 317, 749

Taoso, M., Bertone, G., Meynet, G., Ekström, S. 2008, Physical Review D 78, 123510

van Loon, J.Th., 2006, in Stellar Evolution at Low Metallicity: Mass Loss, Explosions, Cosmology, H.J.G.L.M. Lamers, N. Langer, T. Nugis, K. Annuk, ASP Conf. Series 353, p. 211

Venn, K. A. 1999, ApJ 518, 405

Venn, K. A. Lambert, D. L. 2008, ApJ 677, 572

Venn, K. A., Przybilla, N. 2003, in CNO in the Universe, eds. Charbonnel, Schaerer \& Meynet, ASP Conf Ser. 304, p.20

Vink, J. S., de Koter, A., \& Lamers, H. J. G. L. M. 2001, A\&A 369, 574

von Zeipel, H. 1924, MNRAS 84, 665

Wisniewski, J. P., Bjorkman, K.S. 2006, ApJ 652, 458

Woosley, S. E. 1993, ApJ 405, 273

Woosley, S.E., Bloom, J.S. 2006, ARA\&A 44, 507

Yoon, S.-C., Iocco, F., Akiyama, S. 2008, ApJ 688, L1

Zahn, J.-P. 1992, A\&A 265, 115 